\title{
Evidence based medicine, the Cochrane Collaboration, and the CONSORT statement
}

Evidence based practice uses up to date information to inform decisions on the management of an individual's health problem. ${ }^{1}$ To most, this would seem a basic premise for medical practice, but there has been a backlash against the growth of the evidence based medicine movement. ${ }^{2}$ Some insist that their practice has always been evidence based and that responsible clinicians keep up to date by reading selected journals. But the sheer volume of modern medical publications is such that it is impossible for even experts in an area to be aware of all the relevant literature on the subject. We are inevitably selective, and it is easy for us to select the evidence that confirms what we want to believe. Others fear that clinical freedom is becoming increasingly constrained and that much of the art of medicine is being lost. These fears are unfounded. The evidence base provides us only with the tools we need to be good doctors; the hard earned skills of diagnosis, prognosis, and deciding exactly what balance of risks and benefits apply to the individual patient are enhanced by the knowledge that the options on offer are soundly researched.

The Cochrane Collaboration is a cornerstone of evidence based practice. ${ }^{3}$ Its aim is to provide health professionals and patients alike with easy access to the most up to date evidence of the effectiveness of healthcare interventions. Evidence is summarised and published on the Cochrane Database of Systematic Reviews (CDSR). ${ }^{4}$ Each review includes a description of the efforts to ensure that all relevant published or unpublished evidence is considered for inclusion. The reviews are dynamic and are updated when new evidence emerges or in response to post-publication comments or criticisms. The systematic nature of the process is essential to remove as much bias as possible. The best evidence is provided by randomised controlled trials, in which confounding and bias are minimised by the randomisation process. Controlled observational studies (case-control or cohort) constitute evidence of lower quality because of unknown confounders that cannot be controlled for in the design or analysis. Uncontrolled case series, so prevalent in the ophthalmic literature, rarely provide useful evidence, though outcome studies are useful in auditing quality and assessing clinical effectiveness against guidelines established from an evidence base.

The Cochrane Collaboration concentrates almost exclusively on randomised controlled trials for the time being; it seems logical to concentrate on summarising the best evidence first. A major part of the work of the Cochrane Eyes and Vision Group, registered with the collaboration in April 1997, is the establishment of a register of randomised controlled trials relevant to the field. More than 3000 reports of controlled trials have already been identified, thanks to the work of the Baltimore Cochrane Centre. ${ }^{5}$

At the 1996 Cochrane Colloquium, Iain Chalmers, director of the UK Cochrane Centre in Oxford, challenged all who prevent access to valid evidence to consider the dubious ethics of this behaviour. Strong words, but persuasive in the light of examples where practice is not based on all available evidence; at best, wastage of scarce resources occurs and at worst, wastage of health and sometimes lives.

Two major obstacles exist for those attempting to gain access to the evidence. The first is publication bias, which usually leads to the suppression of studies with negative findings. To deal with this problem, some have proposed the establishment of a journal of negative findings; others suggest a form of positive discrimination to be adopted by investigators and journal editors to overcome their natural desire to publish only the sensational and exciting. Recently, the BMF, the Lancet, and the Annals of Internal Medicine, along with hundreds of other journals, helped to launch the Medical Editors Trial Amnesty, ${ }^{6}$ urging anyone with information about unpublished trials to register them. This register is now included in the Cochrane Library ${ }^{4}$ for use by those designing new studies and those conducting systematic reviews. Unreported trials can be registered by completing and returning an unreported trial registration form, also available in the Cochrane Library.

The second obstacle arises from incomplete or poorly written reports of trials published in the literature. The randomisation process and its concealment are crucial to the validity of any trial. There are many examples in the literature where the science of a trial is compromised by failure to adhere to the randomisation process. Often, when reading a report, one cannot determine exactly what was done in the trial because of ambiguous statements or missing information. To overcome the problem, a leading group of international journal editors have joined forces to agree minimum standards for the reporting of clinical trials, to which all clinical journals are encouraged to formally adhere. This is the CONSORT statement, published in $\mathcal{F} A M A$.

There have been few systematic reviews published in the eye literature; one review on the efficacy of lowering intraocular pressure in chronic glaucoma has had tangible positive outcome. ${ }^{8}$ It has focused the attention of many researchers on seeking ways of providing the necessary evidence and has shown up flaws in the design, execution, analysis, and reporting of many trials. Of more than 120 trials found, only eight could be summarised in a meta-analysis. Some may have been eligible if the CONSORT statement guideline were in place at the time of publication and, perhaps more importantly, at the design stage of the research.

Medical and surgical practice should no longer be a matter of opinion and patients are entitled to expect that what is done to them in the name of orthodox "Western medical practice" is based on scientific research. The challenge is no less great for ophthalmology than it is for any other clinical specialty. The Cochrane Eyes and Vision Group will focus on reviewing areas where there is ambiguity and variation in clinical practice and outcomes, especially where prevention or restoration of sight loss is the goal. The effectiveness of rehabilitation will also be tackled. Any one wishing to contribute to the work of the group, or who wishes to find out more, should contact the authors.

RICHARD WORMALD KATHERINE OLDFIELD 
Glaxo Department of Ophthalmic Epidemiology, Moorfields Eye Hospital and Institute of Ophthalmology, City Road, London EC1V 2PD (email: cevg@ucl.ac.uk)

1 Evidence based medicine: what it is and what it isn't. BMF 1996;312:71-2. 2 Evidence based medicine: Socratic dissent. BMF 1995;310:310-11.

3 The Cochrane Collaboration. BMF 1994;309:969-70.

4 The Cochrane Database of Systematic Reviews in The Cochrane Library (database on disk and CD-ROM.) The Cochrane Collaboration. Oxford: Update Software, 1996, updated quarterly.
5 Scherer R, Dickersin K, Kaplan E, et al. Initiation of a registry of randomized clinical trials in vision research. Invest Ophthalmol Vis Sci 1992; randomized 1323 .

6 Smith R, Roberts I. An amnesty for unpublished trials. BMF 1997;315:622. 7 Begg C, Cho M, Eastwood S, et al. Improving the quality of reporting of randomized controlled trials:the Consort statement. $7 A M A$ 1996;276:637-

8 Rossetti L, Marchetti I, Orzalesi N, et al. Randomized clinical trials on medical treatment of glaucoma. Are they appropriate to guide clinical practice? Arch Ophthalmol 1993;111: 96-103. 